\title{
Caractérisation de la végétation ligneuse et des organisations pelliculaires de surface des agroécosystèmes à différents stades de dégradation de la Commune rurale de Simiri (Niger)
}

\author{
Moussa Mamoudou BOUBACAR ${ }^{1 *}$, Maman Maârouhi INOUSSA ${ }^{1}$, \\ Jean-Marie Karimou AMBOUTA ${ }^{2,3}$, Ali MAHAMANE ${ }^{1,4}$, Aagaard Axelsen JORGEN ${ }^{5}$, \\ Yahou HARISSOU $^{1}$ et Habou RABIOU ${ }^{1}$ \\ ${ }^{1}$ Université Abdou Moumouni de Niamey, Faculté des Sciences, Département de Biologie, \\ Laboratoire Garba Mounkaila, BP 10662 Niamey, Niger. \\ ${ }^{2}$ Université Abdou Moumouni de Niamey, Faculté d'Agronomie, Département des Sciences du Sol, \\ BP 10960 Niamey, Niger. \\ ${ }^{3}$ Université de Tahoua, BP 255 Tahoua, Niger. \\ ${ }^{4}$ Université de Maradi, BP 465, Maradi, Niger. \\ ${ }^{5}$ National Environmental Research Institute, Aarhus University, Vejlsøvej 25; 8600 Silkeborg, Denmark. \\ *Auteur correspondant, E-mail: boubacar_mac@yahoo.fr, Tel :00(227) $97125559 / 90343591$.
}

\section{RESUME}

Cette étude a été réalisée en zone sahélienne dans la Commune rurale de Simiriau, Niger. Elle visait à identifier les stades de dégradation des agroécosystèmes à travers une caractérisation dendrométrique de leurs peuplements ligneux et une description des organisations pelliculaires de surface orientant leur fonctionnement hydrique. Les données dendrométriques et celles relatives aux organisations pelliculaires de surface ont été collectées sur des placettes de $2500 \mathrm{~m}^{2}(50 \mathrm{~m} \times 50 \mathrm{~m})$ le long de quatre transects radiaires à partir du village. Deux stades de dégradation (non dégradé et très dégradé) ont été identifiés dans les agrosystèmes et dans les systèmes sylvopastoraux. Les résultats montrent que pour les deux types d'utilisation des sols (agricole et sylvopastorale), la dégradation se caractérise par, d'une part des faibles valeurs de surface terrière $(0,27 \pm 0,3$ $\mathrm{m}^{2} / \mathrm{ha}$ ), de hauteur moyenne de Lorey $(2,01$ à $2,40 \mathrm{~m})$, de taux de recouvrement des espèces ligneuses $(3,01$ à $6,18 \%)$ et, d'autre part de fréquences élevées en surface productrice de ruissellement (51,3 à 90,54\%). La structure en diamètre des peuplements ligneux des agroécosystèmes dégradés se caractérise par une distribution en « J renversé » avec le paramètre de forme (c) de valeur inférieure à 1 . Ces résultats montrent l'ampleur de la dégradation de ces agroécosystèmes et la nécessité de les restaurer à l'aide des techniques appropriées afin de maitriser le ruissellement et améliorer l'infiltration des eaux de pluies.

(c) 2013 International Formulae Group. All rights reserved.

Mots clés : Paramètres dendrométriques, organisation pelliculaire de surface, agrosystèmes, système sylvopastorale, zone semi-aride, Niger.

\section{INTRODUCTION}

En zone semi-aride, la physionomie de la végétation et les caractéristiques de la surface du sol influencent la variabilité spatiale de l'infiltration et du ruissellement des eaux de pluies (Albergel, 1988). 
L'organisation de la végétation dans cette zone est souvent assimilée à une dégradation du milieu du fait qu'elle se concentre dans des lieux les mieux alimentés en eaux de ruissellement (Ichaou et d'Herbès, 1997; Ludwig et al., 2005). La diminution du couvert végétal suite à la coupe abusive du bois expose le sol aux actions combinées de l'érosion éolienne et hydrique (Moussa et al., 2011). Il en résulte donc un réarrangement des particules du sol qui se traduit par la formation des croûtes de battance en surface, la réduction de la perméabilité du sol, l'augmentation de la compacité et la limitation de l'enracinement (Zougmore et al., 1999). Les conséquences immédiates sont la diminution de la disponibilité en eau conduisant à la baisse des performances dendrométriques des ligneux des écosystèmes cultivés et sylvopastoraux.

$\mathrm{Au}$ Niger, les populations rurales tirent l'essentiel de leur subsistance des ressources naturelles de leur terroir eu égard à la récurrence des années de déficits de production agricole. Cependant, l'exploitation $\mathrm{du}$ bois à travers une coupe abusive non contrôlée à laquelle s'ajoute une forte pression $\mathrm{du}$ bétail, en condition de déficit pluviométrique prolongé, entrainent une dégradation croissante du milieu naturel. Il s'en suit une diminution du couvert végétal et une augmentation des sols nus. Selon Karim et al. (2010), la Commune de Simiri a ainsi connu une hausse significative de la superficie des sols nus au cours des trente dernières années.

La présente étude se fixe pour objectif général de caractériser les agroécosystèmes de la Commune de Simiri. Il s'agit spécifiquement: (a) d'identifier les stades de dégradation des écosystèmes cultivés et sylvopastoraux ; (b) d'évaluer les paramètres dendrométriques de leurs peuplements ligneux; et (c) d'apprécier leur fonctionnement hydrique. Les résultats permettront de proposer des techniques simples de restauration des agroécosystèmes dégradés ainsi identifiés.

\section{MATERIEL ET METHODES Zone d'étude}

La zone d'étude est la Commune rurale de Simiri dont le chef-lieu est situé à $70 \mathrm{~km}$ au Nord de Niamey, la capitale du Niger (Figure 1). Le climat est aride de type Sub-sahélien avec une pluviosité annuelle qui varie entre 400 à 600 mm (Saadou, 1990). Elle est irrégulièrement répartie dans le temps et dans l'espace. La saison des pluies s'étend de juin à septembre. La température moyenne maximale varie de $24{ }^{\circ} \mathrm{C}$ en janvier à $33{ }^{\circ} \mathrm{C}$ en avril avec une amplitude thermique de $9^{\circ} 29$. La toposéquence représentative de la région se caractérise par une succession de quatre grands ensembles typiques des zones de plateaux ferrugineux tropicaux du Sahel, à savoir : le plateau cuirassé, la jupe sableuse, le glacis et les bas-fonds (Ambouta et al., 1996). Des sols squelettiques de types régosols et lithosols formés sur grès argileux du continental terminal s'observent sur les plateaux (Ambouta, 1997). La jupe sableuse et le glacis portent des sols sableux de type ferrugineux tropicaux peu lessivés auxquels font suite des sols alluviaux dans les basfonds. La végétation naturelle est constituée de steppe arbustive sur les glacis et arbustive à arborée dans les bas-fonds tandis qu'une formation contractée soumise à une forte dégradation s'observe sur les plateaux. Les principales espèces ligneuses rencontrées sont Guiera senegalensis, Combretum micranthum et Combretum glutinosum. La strate herbacée est dominée par Mitracarpus scaber, Eragrostistremula et Cenchrus biflorus.

La commune de Simiri totalise 74 villages pour une population estimée à 101627 habitants dont $50,13 \%$ d'hommes et $49,85 \%$ de femmes avec $61,71 \%$ de moins de 25 ans (INS, 2010). Le taux de croissance moyen de la population est estimé à 3,2\% .

Les principales activités de la population de Simiri sont l'agriculture et l'élevage. L'agriculture, pratiquée sur les jupes sableuses, les glacis et les bas-fonds est essentiellement pluviale avec le mil, le sorgho et le niébé comme principales cultures. 
L'élevage du gros bétail et des petits ruminants constitue la seconde activité qui génère des revenus aux populations. Les plateaux constituent, pendant la saison des pluies la seule aire de pâture.

\section{Echantillonnage et collecte des données}

Un échantillonnage par transect radiaire a été réalisé dans le cadre de cette étude. Ainsi, quatre transects ont été installés suivant un gradient d'anthropisation à partir du village de Simiri. Sur chaque transect, des placettes de $2500 \mathrm{~m}^{2}(50 \mathrm{~m} \times 50 \mathrm{~m})$ équidistantes de $500 \mathrm{~m}$ ont été prospectées; soit 38 placettes au total. Les premières placettes de chaque transect ont été installées à $1 \mathrm{~km}$ du village.

Pour la caractérisation de la végétation ligneuse, un inventaire forestier a été réalisé dans chaque placette. Les paramètres dendrométriques tels que le diamètre à $20 \mathrm{~cm}$ au-dessus du sol, la hauteur totale, et les deux diamètres du houppier ont été mesurés (Morou, 2010). Les mesures ont concerné les individus au diamètre supérieur ou égal à 4 $\mathrm{cm}$ (SUN, 2008). Les tiges de diamètre inférieur à $4 \mathrm{~cm}$ ont été considérées comme des régénérations et leur nombre a été évalué. Les organisations pelliculaires de surface (OPS) ont été décrites dans chacune des placettes selon la méthode proposée par Ambouta et al. (1996). Selon cette méthode, un réseau rectangulaire constitué de 32 points répartis de façon régulière avec une maille carrée de $8 \mathrm{~m}$ de côté a été placé (Figure 2). Sur $20 \mathrm{~cm}$ autour de chaque point, le type de croûte dominant a été noté selon la typologie de Casenave et Valentin (1989) élargie aux croûtes biologiques. La grille est installée sur une partie la plus représentative possible des états de surface de la placette. Ainsi, les croûtes structurales (ST), les croûtes d'érosion (ERO), les croûtes gravillonnaires (GRA), les croûtes de ruissellement (RUI) et les croûtes biologiques (BIO) ont été considérées comme des organisations pelliculaires de surface contribuant à la formation des surfaces productrices de ruissellement (SPR). Par contre, les croûtes de décantation (DEC) et les croûtes de dessiccation (DES) ont été retenues comme des organisations pelliculaires de surface contribuant à la formation des surfaces de stockage et d'infiltration (SSI) (Ichaou, 2000).

\section{Analyse des données \\ Paramètres dendrométriques}

Dans chaque placette, les paramètres suivant ont été calculés :

- $\quad$ Le taux de recouvrement $(R)$ des espèces ligneuses en pourcentage $(\%)$ est obtenu par la formule suivante :

$$
\mathrm{R}(\%)=\frac{\mathrm{r} \times 100}{\mathrm{~S}} \quad \text { avec } \quad r=\frac{\Pi}{4} \sum_{i=1}^{n} d i^{2}
$$

$r=$ recouvrement de l'ensemble des individus de la placette $\left(\mathrm{m}^{2}\right) ; d i=$ diamètre moyen du houppier de l'individu i (m); s = superficie de la placette $\left(\mathrm{m}^{2}\right)$.

- $\quad$ La densité en tiges $(N)$ exprimée en tiges par hectare (Tiges/ha) est déterminée par le nombre total de tiges dans chaque placette suivant la formule :

$\mathrm{N}=\frac{\mathrm{n}}{\mathrm{s}}$

$n=$ nombre total de tiges inventoriées dans la placette et $s=$ superficie de la placette en hectare.

- La densité en régénération $(N r)$ est exprimée en tiges par hectare (Tiges/ha) par la formule suivante :

$$
\mathrm{N} r=\frac{n r}{\mathrm{~s}}
$$

$n r=$ nombre total de tiges de diamètre inférieur à $4 \mathrm{~cm}$ recensées dans la placette et $\mathrm{s}$ $=$ superficie de la placette en hectare.

- Le diamètre moyen $(D g)$ exprimé en centimètre $(\mathrm{cm})$ est déterminé à l'échelle de chaque placette par la formule : $n=$ nombre total de tiges rencontrées dans la placette et $d i=$ diamètre de la tige $\mathrm{i}(\mathrm{cm})$. 


$$
\mathrm{D}_{\mathrm{g}}=\sqrt{\frac{1}{\mathrm{n}} \sum_{\mathrm{i}=1}^{\mathrm{n}} \mathrm{d}_{\mathrm{i}}^{2}}
$$

La surface terrière globale $(G)$, exprimée en $\mathrm{m}^{2} /$ ha, est donnée par la formule :

$$
\mathrm{G}=\frac{\pi}{40000 \mathrm{~s}} \sum_{\mathrm{i}=1}^{\mathrm{n}} \mathrm{d}_{\mathrm{i}}^{2}
$$

$s=$ superficie de la placette en hectare et $d i=$ diamètre de la tige $\mathrm{i}(\mathrm{cm})$.

La hauteur moyenne de Lorey $\left(H_{L}\right)$ exprimé en mètre (m) est la hauteur moyenne des individus pondérés à leur surface terrière (Philip, 2002). La formule est la suivante :

$$
\mathrm{H}_{\mathrm{L}}=\frac{\sum_{\mathrm{i}=1}^{\mathrm{n}} \mathrm{g}_{\mathrm{i}} \mathrm{h}_{\mathrm{i}}}{\sum_{\mathrm{i}=1}^{\mathrm{n}} \mathrm{g}_{\mathrm{i}}} \quad \text { avec } \quad \mathrm{g}_{\mathrm{i}}=\frac{\pi}{4} \mathrm{~d}_{\mathrm{i}}^{2}
$$

Où gi et $h i$ sont respectivement la surface terrière et la hauteur totale de l'individu i.

- Pour la structure en classes de diamètre des groupes identifiés, les individus de diamètre supérieur à $4 \mathrm{~cm}$ ont été répartis en 10 classes de diamètre de $6 \mathrm{~cm}$ d'amplitude. La structure de chaque groupe a été ajustée au modèle de Weibull, choisi pour sa grande flexibilité (Bonou et al., 2009). La fonction de densité de probabilité de la distribution de Weibull, se présente sous la forme de l'équation suivante (Rondeux, 1999) :

$$
f(x)=\frac{c}{b}\left(\frac{x-a}{b}\right)^{c-1} \exp \left[-\left(\frac{x-a}{b}\right)^{c}\right]
$$

Où $\mathrm{x}=\mathrm{le}$ diamètre de la tige $\mathrm{i} ; \mathrm{a}=\mathrm{le}$ paramètre de position $(4 \mathrm{~cm}) ; \mathrm{b}=1 \mathrm{e}$ paramètre d'échelle ou de taille; $c=1 e$ paramètre de forme lié à la structure observée. La comparaison des structures diamétriques des peuplements des différents groupes a été réalisée sur la base du paramètre de forme (Bonou et al., 2009). Une analyse log-linéaire a été effectuée grâce au logiciel SAS (SAS Inc, 1999) pour tester l'adéquation de la structure observée à la distribution théorique de Weibull (Caswell, 2001). L'hypothèse de l'adéquation entre les deux distributions (théorique et observée) a été acceptée si la valeur de la probabilité du test est supérieure à 0,05 .

Calcul des fréquences des organisations pelliculaires de surface (OPS)

Dans chaque placette, les fréquences suivantes ont été calculées :

- La fréquence de chaque type de croûteexprimée en pourcentage (\%) a été calculée à travers la formule suivante :

$\operatorname{Fr}(i)=\frac{n \times 100}{32}$

$i$ : type de croûte; $n:$ nombre total de points inventoriés pour le type de croûte considéré.

- La fréquence en SPR: elle est expriméeen pourcentage (\%) et calculée par la formule suivante :

$$
F r(S P R)=F r(S T)+F r(E R \partial)+F r(G R A)+F r(R U I)+F r(B I O)
$$

- $\quad$ La fréquence en SSI : elle est également exprimée en pourcentage (\%) et a été calculée par la formule suivante :

$$
\operatorname{Fr}(S S I)=\operatorname{Fr}(D E C)+\operatorname{Fr}(D E S)
$$

\section{Analyse multivariée}

L'identification des stades de dégradation des agroécosystèmes a été effectuée à travers une analyse en composante principale (ACP) sur une matrice 13 paramètres et 38 relevés. Les paramètres dendrométriques et environnementaux ayant des coefficients de corrélation de Pearson supérieurs à la valeur absolue de 0,5 avec les deux premiers axes du plan factoriel ont été retenus comme critères d'appréciation de chaque stade de dégradation des agroécosystèmes. Pour comparer les stades de dégradation d'un même type d'utilisation des sols en fonction des paramètres dendrométriques et environnementaux, un test non paramétrique de Kruskal-Wallis a été réalisé. Le logiciel JMP 9 (SAS, 2010) a été utilisé pour l'ensemble des traitements statistique. 


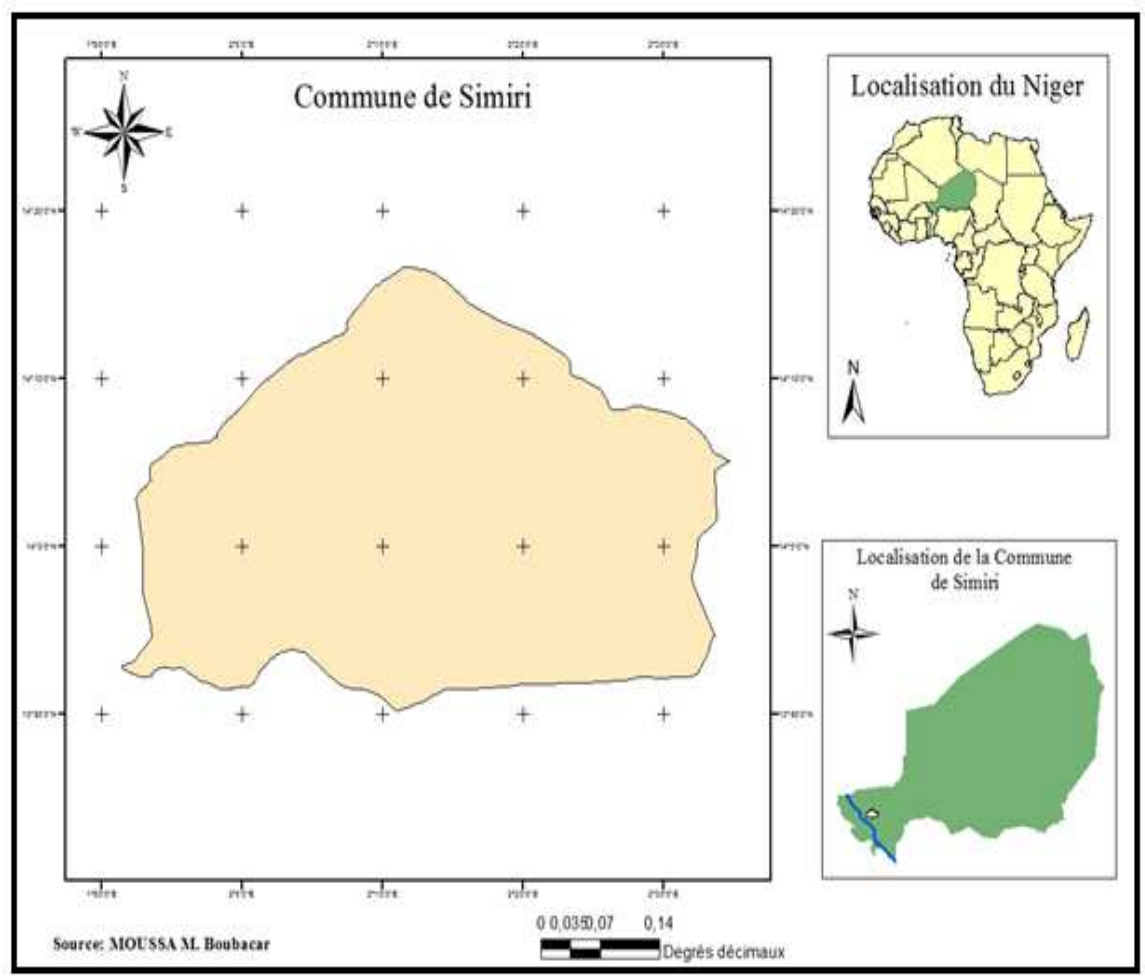

Figure 1: Localisation de la Commune de Simiri.

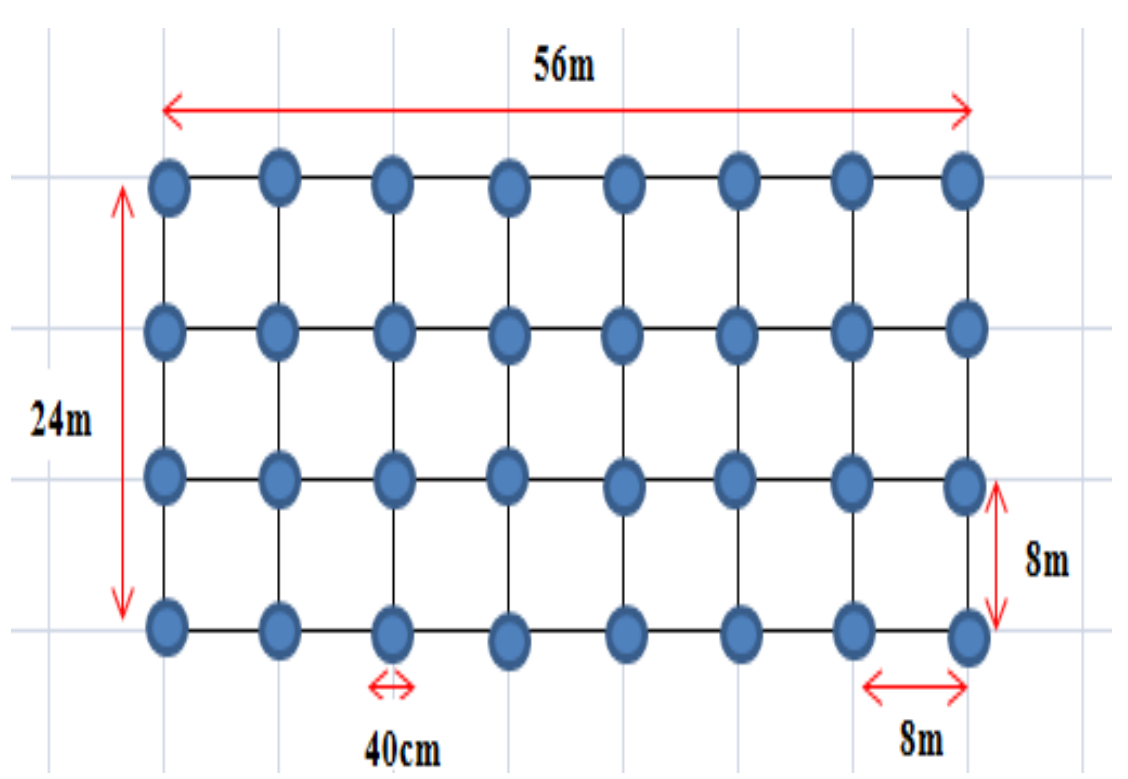

Figure 2 : Grille de relevé des organisations pelliculaires de surface. 


\section{RESULTATS}

\section{Identification des stades de dégradation}

La Figure 3 montre que les deux premiers axes expliquent $75,9 \%$ des informations avec $47,5 \%$ pour l'axe 1 et $28,4 \%$ pour l'axe 2. Par conséquent seul ces deux axes ont été retenus pour l'identification des stades de dégradation des agroécosystèmes.

Le Tableau 1 fait ressortir les coefficients de corrélation de Pearson entre les paramètres dendrométriques et environnementaux avec les deux premiers axes. Il ressort que :

- L'axe 1 concentre les informations relatives aux paramètres tels que les agrosystèmes des bas-fonds, les systèmes sylvopastoraux des plateaux, les surfaces productrices de ruissellement (SPR), les surfaces de stockage et d'infiltration (SSI), le diamètre moyen, la surface terrière et la hauteur moyenne de Lorey. Ainsi, le groupe G3 situé du côté positif de l'axe regroupe les relevés des bas-fonds avec d'une part des fréquences élevées en surface de stockage et d'infiltration et d'autre part des valeurs élevées en individus de diamètre moyen, de surface terrière et de hauteur moyenne de Lorey. G3 définit donc un groupe d'agrosystèmes de bas-fonds non dégradés. Par contre, le groupe G1 situé du côté négatif de l'axe 1 regroupe des relevés des plateaux avec des fréquences élevées en surface productrice de ruissellement et des valeurs faibles en individus de diamètre moyen, de surface terrière et de hauteur moyenne de Lorey. G1 est un groupe composé d'aires sylvopastorales sur les plateaux très dégradés.

- L'axe 2 est corrélé aux agrosystèmes des glacis, aux systèmes sylvopastoraux des plateaux, à la surface terrière, à la densité en tiges, au taux de recouvrement et à la densité de régénération. Ainsi, le groupe G2 situé du côté positif de cet axe est une aire sylvopastorale sur plateaux avec des individus ayant des valeurs élevées de surface terrière, de densité en arbres, de taux de recouvrement et de densité de régénération. Il peut être défini comme un groupe des écosystèmes sylvopastoraux des plateaux non dégradés. Contrairement à G2, le groupe G4 est une zone agricole des glacis présentant des individus avec de faible valeur de surface terrière, de densité en tiges, de taux de recouvrement en arbres et de densité de régénération. Ce groupe peut être identifié comme celui des agrosystèmes des glacis très dégradés.

\section{Caractéristiques dendrométriques}

Le Tableau 2 donne une comparaison des moyennes des paramètres dendrométriques en fonction des stades de dégradation dans chaque agroécosystème. Il ressort de l'analyse globale de ce tableau qu'il existe une différence très significative $(\mathrm{P}<0,001)$ entre les différents stades de dégradation pour la surface terrière $(G)$, la hauteur moyenne de Laurey (HL) et le taux de recouvrement (R). Ainsi, les systèmes sylvopastoraux de plateaux très dégradés $(\mathrm{G} 1)$ se caractérisent par des valeurs faibles de surface terrière $\left(0,27 \pm 0,3 \mathrm{~m}^{2} / \mathrm{ha}\right)$, de hauteur moyenne de Lorey $(2,01 \pm 0,77 \mathrm{~m})$, de densité en tiges (1205 \pm 1589 tiges/ha), de densité en régénération (1124 \pm 1503 tiges/ha), de taux de recouvrement $(6,18 \pm 9,11 \%)$ par rapport aux systèmes de plateaux non dégradés (G2). Par ailleurs dans les agrosystèmes, les glacis très dégradés (G4) se distinguent par des valeurs faibles en diamètre moyen $(7,24 \pm 3,58 \mathrm{~cm})$, en surface terrière $\left(0,26 \pm 0,21 \mathrm{~m}^{2} / \mathrm{ha}\right)$, en hauteur moyenne de Lorey $(2,40 \pm 0,75 \mathrm{~m})$ et en taux de recouvrement ligneux $(3,01 \pm 0,90 \%)$ par rapport aux bas-fonds non dégradés (G3). 
Les structures en diamètre des différents stades de dégradation ont été réalisées avec surimposition de la distribution de Weibull (Figure 4). Les résultats de l'analyse log-linéaire montrent que les distributions observées des différents stades de dégradation des agroécosystèmes s'ajustent globalement avec les distributions de Weibull respectives $(\mathrm{P}>0,05)$. Pour l'ensemble des groupes (G1, G2 et G4), la structure en diamètre des individus ligneux est caractéristique d'une distribution en «J renversé » avec le paramètre de forme (c) de valeur inférieure à 1 . On remarque également une absence totale de tiges de diamètre supérieur à $22 \mathrm{~cm}$ dans les systèmes sylvopastoraux très dégradés (G1).

\begin{abstract}
Aptitude des agroécosystèmes au ruissellement et à l'infiltration

La Figure 5 montre que les agroécosystèmes très dégradés (G1 et G4) sont caractérisés par les valeurs les plus élevées de surfaces productrices de ruissellement $\quad(90,54 \%$ et $51,3 \%$ respectivement) avec une probabilité de $\mathrm{P}=0,001$. $\mathrm{G} 2$ et $\mathrm{G} 3$ sont les groupes qui enregistrent les valeurs les plus élevées en surface de stockage et d'infiltration $(79,9 \%$ et $97,8 \%$ respectivement) avec une probabilité de $\mathrm{P}=0,001$.
\end{abstract}

Tableau 1 : Coefficients de corrélation de Pearson entre les paramètres dendrométriques, environnementaux et les deux premiers axes du plan factoriel.

\begin{tabular}{llll}
\hline Paramètres & & Axe 1 & Axe 2 \\
\hline \multirow{4}{*}{ Dendrométriques } & $\mathrm{Dg}$ & 0,895 & 0,119 \\
& $\mathrm{G}$ & 0,666 & 0,643 \\
& $\mathrm{~N}$ & $-0,314$ & 0,611 \\
& $\mathrm{Nr}$ & $-0,330$ & 0,585 \\
& $\mathrm{H}_{\mathrm{L}}$ & 0,868 & 0,281 \\
& $\mathrm{R}$ & 0,247 & 0,867 \\
\hline \multirow{3}{*}{ Environnementaux } & $\mathrm{SPR}$ & $-0,849$ & $-0,321$ \\
& $\mathrm{SSI}$ & 0,849 & 0,321 \\
& $\mathrm{Plateau}$ & $-0,744$ & 0,569 \\
& Glacis & $-0,054$ & $-0,733$ \\
& Bas-fond & 0,898 & 0,088 \\
& Sylvopastorale & $-0,744$ & 0,569 \\
& Agricole & 0,744 & $-0,569$
\end{tabular}

$\mathrm{Dg}$ : diamètre moyen ; $\mathrm{G}$ : surface terrière globale ; $\mathrm{N}:$ Densité en tiges ; $\mathrm{Nr}$ : densité en régénération ; $\mathrm{H}_{\mathrm{L}}$ : Hauteur moyenne de Lorey ; R : taux de recouvrement ; SPR : surface productrice de ruissellement ; SSI : surface de stockage et d'infiltration. 
Tableau 2 : Comparaison des moyennes (m) des paramètres dendrométriques en fonction des stades de dégradation dans chaque agroécosystème.

\begin{tabular}{|c|c|c|c|c|c|c|c|c|c|c|}
\hline \multirow{3}{*}{$\begin{array}{l}\text { Paramètres } \\
\text { dendrométriques }\end{array}$} & \multicolumn{5}{|c|}{ Systèmes sylvopastoraux } & \multicolumn{5}{|c|}{ Agrosystèmes } \\
\hline & \multicolumn{2}{|c|}{ G1 $(n=11)$} & \multicolumn{2}{|c|}{ G2 $(n=7)$} & \multirow[t]{2}{*}{$\mathbf{P}$} & \multicolumn{2}{|c|}{ G3 $(n=10)$} & \multicolumn{2}{|c|}{ G4 (n=10) } & \multirow[t]{2}{*}{$\mathbf{P}$} \\
\hline & $\mathbf{m}$ & $\sigma$ & $\mathbf{m}$ & $\sigma$ & & $\mathbf{m}$ & $\sigma$ & $\mathbf{m}$ & $\sigma$ & \\
\hline $\operatorname{Dg}(\mathrm{cm})$ & $5,91^{\mathrm{a}}$ & 1,35 & $10,07^{\mathrm{a}}$ & 4,54 & 0,260 & $30,26^{\mathrm{b}}$ & 7,17 & $7,24^{\mathrm{a}}$ & 3,58 & 0,000 \\
\hline $\mathrm{G}\left(\mathrm{m}^{2} \times \mathrm{ha}^{-1}\right)$ & $0,27^{\mathrm{a}}$ & 0,30 & $3,48^{\mathrm{b}}$ & 1,57 & 0,000 & $3,83^{\mathrm{b}}$ & 1,11 & $0,27^{\mathrm{a}}$ & 0,21 & 0,000 \\
\hline $\mathrm{H}_{\mathrm{L}}(\mathrm{m})$ & $2,01^{\mathrm{a}}$ & 0,77 & $3,87^{\mathrm{b}}$ & 1,44 & 0,005 & $6,40^{\mathrm{c}}$ & 0,97 & $2,40^{\mathrm{a}}$ & 0,75 & 0,000 \\
\hline $\mathrm{N}\left({\left.\text { Tiges } \times \mathrm{ha}^{-1}\right)}^{-1}\right.$ & $1205^{\mathrm{a}}$ & 1589 & $8150^{\mathrm{b}}$ & 3518 & 0,001 & $591^{\mathrm{a}}$ & 522 & $960^{\mathrm{a}}$ & 390 & 0,075 \\
\hline $\operatorname{Nr}\left(\right.$ Tiges $\left.\times \mathrm{ha}^{-1}\right)$ & $1124^{\mathrm{a}}$ & 1503 & $7378^{\mathrm{b}}$ & 3327 & 0,001 & $524^{\mathrm{a}}$ & 483 & $900^{\mathrm{a}}$ & 357 & 0,045 \\
\hline $\mathrm{R}(\%)$ & $6,18^{\mathrm{a}}$ & 9,11 & $40,56^{\mathrm{c}}$ & 11,80 & 0,000 & $20,85^{\mathrm{b}}$ & 9,91 & $3,01^{\mathrm{a}}$ & 0,90 & 0,000 \\
\hline
\end{tabular}
plateaux non dégradés ; $\mathrm{G} 3$ : groupe des agrosystèmes de bas-fonds non dégradés ; G4 : groupe des agrosystèmes de glacis très dégradés ; $\mathrm{m}:$ moyenne ; $\sigma$ : écart type $; \mathrm{P}$ : probabilité ; Dg : diamètre moyen ; $\mathrm{G}$ : surface terrière globale ; $\mathrm{N}$ : Densité en tiges ; $\mathrm{Nr}$ : densité en régénération; $\mathrm{H}_{\mathrm{L}}$ : Hauteur moyenne de Lorey; $\mathrm{R}$ : taux de recouvrement. Sur chaque ligne, les valeurs accompagnées par des lettres différentes sont significativement différentes au seuil de probabilité $\alpha=0,05$.

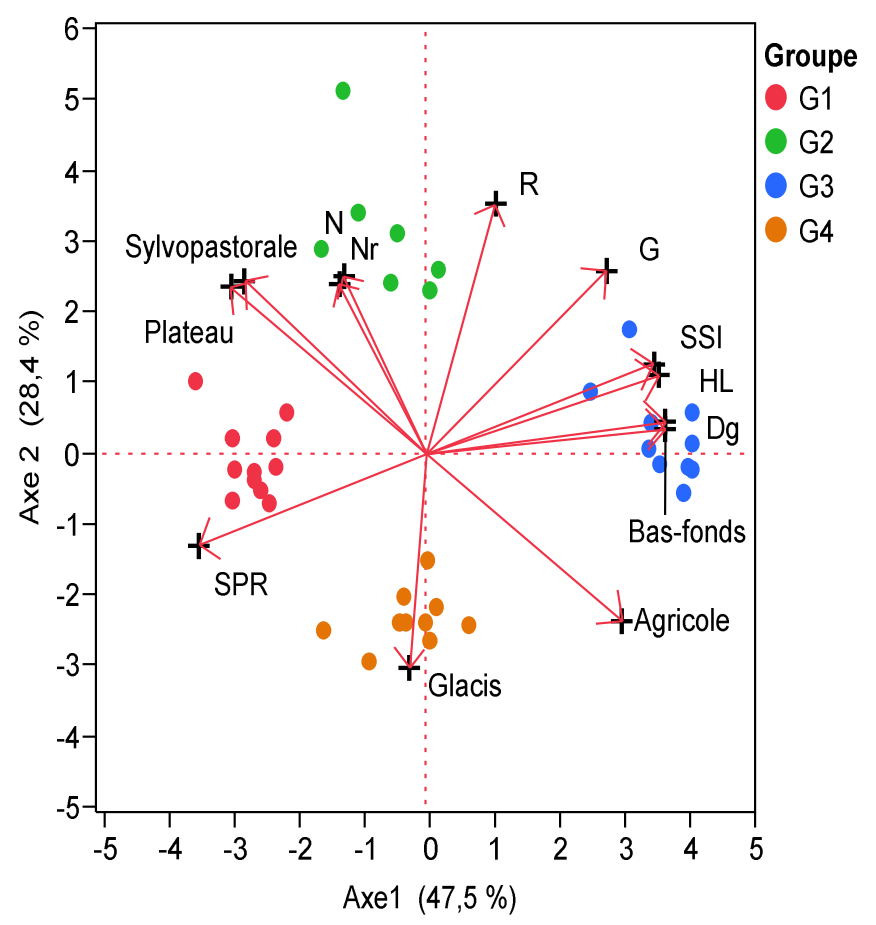

Figure 3: Projection des relevés dans le système d'axes de l'Analyse en Composante Principale $(A C P) . D g$ : diamètre moyen ; $\mathrm{G}$ : surface terrière globale ; $\mathrm{N}$ : Densité en tiges ; $\mathrm{Nr}$ : densité en régénération ; $\mathrm{H}_{\mathrm{L}}$ : Hauteur moyenne de Lorey ; R : taux de recouvrement ; SPR : surface productrice de ruissellement ; SSI : surface de stockage et d'infiltration; G1: groupe des systèmes sylvopastoraux de plateaux très dégradés ; G2 : groupe des systèmes sylvopastoraux de plateaux non dégradés; G3: groupe des agrosystèmes de bas-fonds non dégradés ; G4 : groupe des agrosystèmes de glacis très dégradés. 


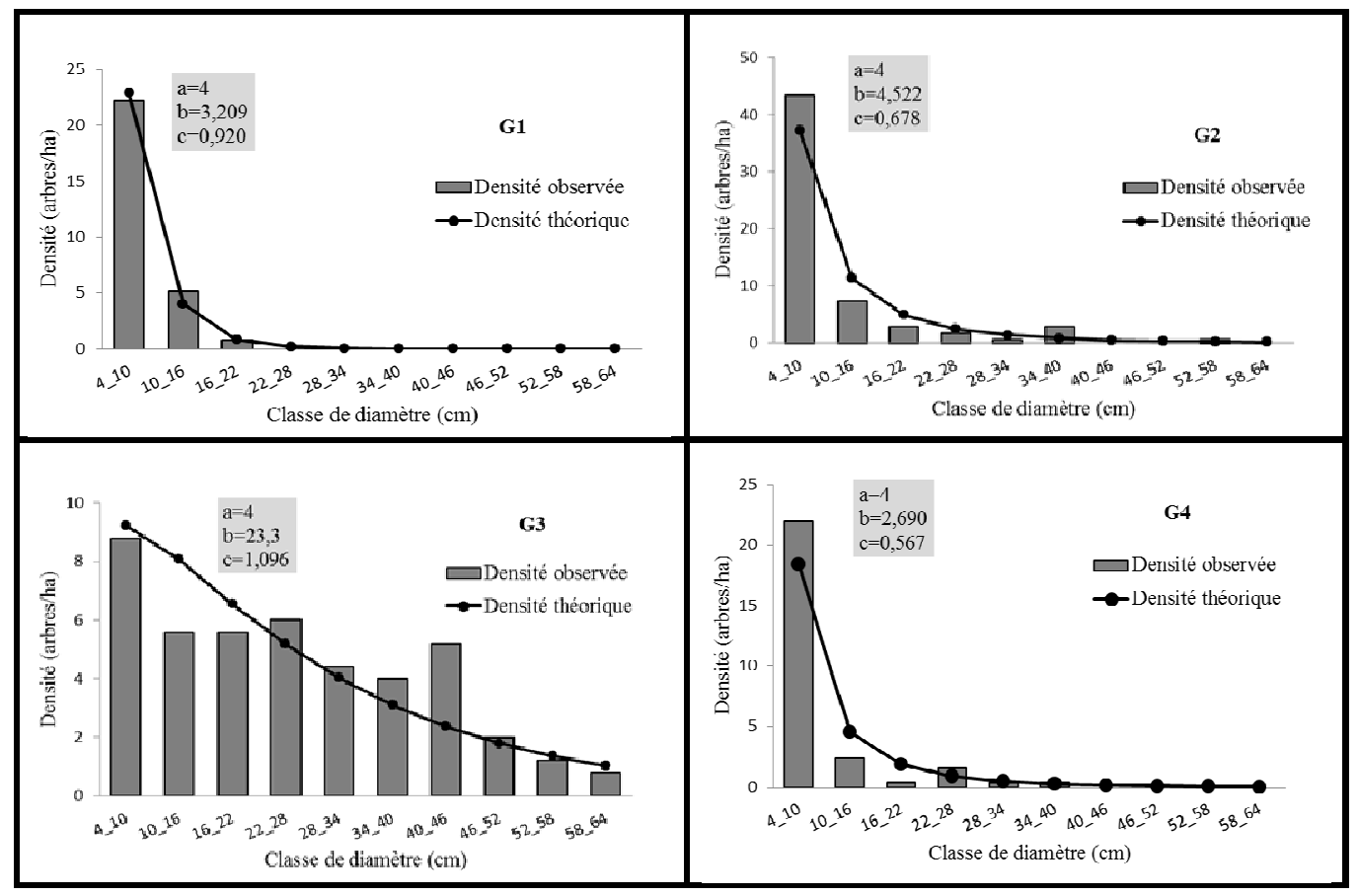

Figure 4: Structure en diamètre des stades de dégradation (G1, G2, G3 et G4). G1: groupe des systèmes sylvopastoraux de plateaux très dégradés; G2 : groupe des systèmes sylvopastoraux de plateaux non dégradés ; G3 : groupe des agrosystèmes de bas-fonds non dégradés ; G4 : groupe des agrosystèmes de glacis très dégradés.

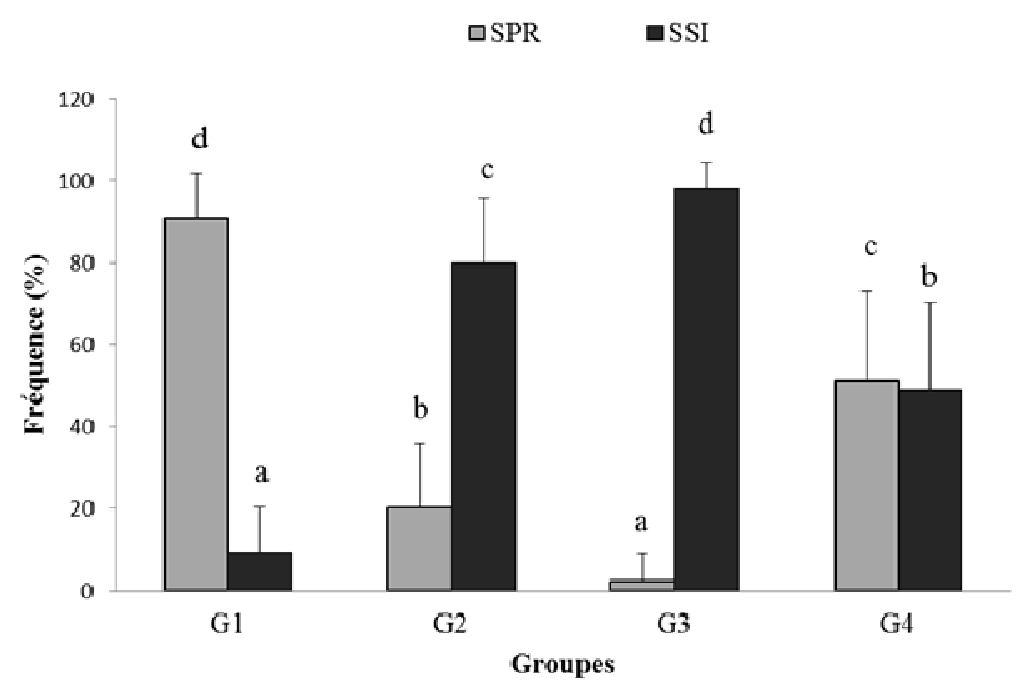

Figure 5: Fréquence (\%) en surface productrice de ruissellement (SPR) et en surface de stockage et d'infiltration (SSI) des groupes (G1, G2, G3 et G4). SPR : surface productrice de ruissellement ; SSI : surface de stockage et d'infiltration ; G1 : groupe des systèmes sylvopastoraux de plateaux très dégradés; G2 : groupe des systèmes sylvopastoraux de plateaux non dégradés; G3 : groupe des agrosystèmes de bas-fonds non dégradés ; G4 : groupe des agrosystèmes de glacis très dégradés. Pour chaque organisation pelliculaire de surface, les groupes accompagnés par des lettres différentes sont significativement différents au seuil de probabilité $\alpha=0,05$. 


\section{DISCUSSION}

\section{Les écosystèmes sylvopastoraux}

Sur les plateaux de la Commune de Simiri se développent quelques reliques de formation contractée communément appelée «Brousse tigrée », non dégradée (G2) à vocation sylvopastorale. Ils se caractérisent par des taux élevés en surface de stockage et d'infiltration $(79,85 \%)$. En effet, les surfaces favorables au ruissellement $(20,14 \%)$ jouent le rôle d'impluvium pour alimenter en eaux de ruissellement la végétation contractée des zones d'infiltration (Galle et al., 1997). Les individus ligneux qui y vivent se caractérisent par des valeurs élevées de surface terrière, de densité en tiges, de hauteur moyenne et de taux de recouvrement. La forte densité de régénération $(7378 \pm 3327$ tiges/ha) est liée à la forte capacité de régénération par voie végétative de Combretum micranthum, espèce clef de voûte de cet agroécosystème (Couteron, 2001 ; Gilad et al., 2004 ; Diouf et al., 2010). En effet, cette espèce développe des tiges adventives à partir du système racinaire (drageon) ou du système aérien (marcotte) lui permettant de subsister dans des conditions défavorables (Bellefontaine, 2005). En plus, elle entraine une amélioration de la structure du sol ou de la teneur en matière organique de son biotope (Hiernaux et al., 1999; Barbier et al., 2006). Quant à la structure en diamètre des individus ligneux, elle donne une valeur du paramètre de forme (c) inférieure à 1, caractéristique d'un peuplement en pleine régénération. Cependant, les pressions anthropiques de la population qui vit en grande partie des revenues de l'exploitation du bois entrainent une diminution de la densité de tiges à l'hectare (1205 \pm 1589$)$. Cela s'observe à travers la structure en classes de diamètre qui fait ressortir une faible densité des arbres pour les classes de diamètre compris entre 4 et 16 $\mathrm{cm}$ et une absence totale de tiges de diamètre supérieur à $22 \mathrm{~cm}$. Les faibles valeurs de surface terrière $\left(0,27 \pm 0,3 \mathrm{~m}^{2} / \mathrm{ha}\right)$ et de taux de recouvrement $(6,18 \pm 9,11 \%)$ sont des indicateurs de la disparition progressive de la couverture végétale. La faible couverture sableuse des plateaux latéritiques de ces agroécosystèmes est alors exposée aux actions combinées de l'érosion éolienne et hydrique favorisant la formation des croûtes à la surface du sol (Moussa et al., 2011). Selon Ambouta et al. (1996), la croûte d'érosion est un état de surface qui influence de façon significative les propriétés du sol. La formation de ces croûtes débute par la dégradation de la structure du sol à travers la destruction des agrégats. Cette réorganisation structurale entraine la compaction de la surface du sol sous l'effet des gouttes de pluie provoquant une obturation des pores permettant l'infiltration. Il s'en suit une augmentation du ruissellement $(90,54 \%)$ et une diminution de l'infiltration $(9,45 \%)$. Ces résultats corroborent ceux de Yaméogo et al. (2009) obtenus dans les écosystèmes dégradés de la forêt classée de Kuinima en zone soudanienne au Burkina Faso.

\section{Les agrosystèmes}

Dans les agrosystèmes, la géomorphologie et les états de surface jouent un rôle déterminant dans la fertilité des sols (Mahamane et al., 2007). Les bas-fonds du fait de leur position topographique bénéficient d'un supplément d'eau et de la présence de certaines espèces fixatrices d'azote améliorant ainsi la fertilité des sols. La disponibilité de l'eau, la texture sablo-argileuse du sol et la profondeur assez élevée des sols des basfonds permettent le développement d'une végétation arbustive relativement dense. Elle se caractérise par des individus de diamètre moyen élevé $(30,26 \pm 7,17 \mathrm{~cm})$, de surface terrière importante $\left(3,83 \pm 1,11 \mathrm{~m}^{2} / \mathrm{ha}\right)$, de hauteur moyenne de Lorey élevée $(6,4 \pm 0,97$ 
m) et un fort recouvrement des arbustes $(20,85 \pm 9,91 \%)$. Par contre, sur les glacis, les valeurs faibles de diamètre moyen $(7,24 \pm 3,58$ $\mathrm{cm})$, de surface terrière $\left(0,26 \pm 0,21 \mathrm{~m}^{2} / \mathrm{ha}\right)$ et de taux de recouvrement $(3,01 \pm 0,90 \%)$ peuvent s'expliquer par la faible densité en tiges dans ces agrosystèmes. Le taux de recouvrement est un indicateur assez important de la dégradation des sols du fait qu'il détermine le degré de sensibilité du sol à la pluie (Robert, 1992). La texture sableuse fine et la faible teneur en matière organique des sols de ces agrosystèmes contribuent également à la formation des croûtes d'érosion à la surface du sol (Ambouta, 1994). Cela se traduit par des fréquences assez élevées $(51,3 \%)$ en surfaces productrices de ruissellement dans ces agrosystèmes. Cependant, des études spécifiques sur la fertilité physico-chimique des sols des différents états de surface qui n'ont pas pu être réalisées dans le cadre de ce travail devront être envisagées.

\section{Conclusion}

Cette étude a permis d'identifier deux stades de dégradation des agroécosystèmes de la Commune rurale de Simiri pour chaque type d'utilisation des sols. Le premier stade concerne les agroécosystèmes non dégradés sylvopastoraux des plateaux et les agrosystèmes des bas-fonds. Ils se caractérisent d'une part par des valeurs importantes de surface terrière $(3,48$ à 3,83 $\mathrm{m}^{2} /$ ha), de hauteur moyenne $(3,87$ à $6,40 \mathrm{~m})$, de taux de recouvrement des espèces ligneuses $(20,85$ à 40,56\%) et, d'autre part par des fréquences élevées en surfaces de stockage et d'infiltration des eaux de pluies (79,85 à 97,8\%). Le deuxième stade concerne les agroécosystèmes très dégradés sylvopastoraux des plateaux et les agrosystèmes des glacis qui se définissent d'une part par des faibles valeurs de surface terrière $\left(0,27 \pm 0,3 \mathrm{~m}^{2} / \mathrm{ha}\right)$, de hauteur moyenne de Lorey $(2,01$ à $2,40 \mathrm{~m})$, de taux de recouvrement des espèces ligneuses (3,01 à $6,18 \%$ ) et, d'autre part par des fréquences élevées en surface productrice de ruissellement (51,3 à 90,54\%). Ces résultats montrent la nécessité de tester sur ces agroécosystèmes très dégradés des techniques simples mais efficaces de restauration. Des techniques telles que les zaï, les demi-lunes, les cordons pierreux et le paillage peuvent être réalisées en zone agricole et des banquettes sur les plateaux à vocation sylvopastorale. Ces techniques permettront de maîtriser le ruissellement et d'améliorer l'infiltration des eaux de pluies dans ces agroécosystèmes dégradés.

\section{REMERCIEMENTS}

Nous remercions toutes les personnes ressources qui ont contribuées à l'élaboration de ce document. Nos remerciements vont également au programme UNDESERT qui a financé nos différentes activités.

\section{REFERENCES}

Albergel J. 1988. Genèse et Prédétermination des Crues au Burkina Faso. Du $m^{2}$ au $\mathrm{km}^{2}$. Etude des Paramètres Hydrologiques et de leur Evolution. Coll. Etudes et Thèses, ORSTOM : Paris.

Ambouta JMK. 1994. Etude des facteurs de formation d'une croûte d'érosion et de ses relations avec les propriétés internes d'un sol sableux fin au Sahel. Thèse présentée à l'Université Laval, Québec, 86p.

Ambouta JMK, Valentin C, Laverdière MR. 1996. Jachère et croûtes d'érosion au sahel. Sécheresse, 7: 269-75.

Ambouta JMK. 1997. Définition et caractérisation des structures de végétation contractée au sahel: cas de la brousse tigrée de l'ouest nigérien. In Fonctionnement et Gestion des 
Ecosystèmes Forestiers Contractés Sahéliens, d'Herbès JM, Ambouta JMK, Peltier R (éds). John Libbey Eurotext : Paris; 41-57.

Barbier N, Couteron P, Lejoly J, Deblauwe V, Lejeune O. 2006. Self-organized patterning as fingerprint of climate and human impact on semi-arid ecosystems. J. Ecol., 94(3): 537-547.

Bellefontaine R. 2005. Pour de nombreux ligneux, la reproduction sexuée n'est pas la seule voie : analyse de 875 cas - Texte introductif, tableau et bibliographie. Sécheresse - Revue Electronique, 3. http://www.secheresse.info/article.php3? id_article $=2344$

Bonou W, GléléKakaï R, Assogbadjo AE, Fonton HN, Sinsin B. 2009. Characterisation of Afzelia africana Sm. Habitat in Lama forest reserve of Benin. Forest Ecology and Management, 258: 1084-1092.

Casenave A, Valentin C. 1989. Les Etats de Surface de la Zone Sahélienne. Influence sur l'Infiltration. Orstom Editions : Paris.

Caswell H. 2001. Matrix Population Models : Construction Analysis and Interpretation $\left(2^{\text {nd }} \quad e d n\right)$. Sinauer Associates: Massachusetts; 12 p.

Couteron P. 2001. Using spectral analysis to confront distribution of individual species with an overall pattern. Plant Ecol., 156(2): 229-243.

Diouf A, Barbier N, Mahamane A, Lejoly J, Saadou M, Bogaert J. 2010. Caractérisation de la structure spatiale des individus ligneux dans une "Brousse tachetée" au Sud-ouest du Niger. Canadian Journal of Forest Research, 40: $827-835$.

Galle S, Seghieri J, Mounkaila H. 1997. Fonctionnement hydrique et biologique à l'échelle locale. Cas d'une brousse tigrée au Niger. In Fonctionnement et Gestion des Ecosystèmes Forestiers Contractés Sahéliens, d'Herbès JM, Ambouta JMK, Peltier R (éds). John Libbey Eurotext: Paris; 105-118.

Gilad E, von hardenberg J, Provenzale A, Shachak M, Meron E. 2004. Ecosystem engineers: from pattern formation to habitat creation. Phys. Rev. Let., 93(9).

Hiernaux P, Bielders CL, Valentin C, Bationo A, Fernandez-Rivera S. 1999. Effects of livestock grazing on physical and chemical properties of sandy soil in Sahelian ranglands. J. Arid Environ., 41(3): 231-245.

Ichaou A, d'Herbès JM. 1997. Productivité comparée des formations structurées et non structurées dans le Sahel nigérien. Conséquences pour la gestion forestière. In Fonctionnement et Gestion des Ecosystèmes Forestiers Contractés Sahéliens, d'Herbès JM, Ambouta JMK, Peltier R (éds). John Libbey Eurotext: Paris ; 119-130.

Ichaou A. 2000. Dynamique et productivité des structures forestières contractées des plateaux de l'Ouest nigérien. Thèse Ecologie Végétale Tropicale, Université Sabatier, Toulouse, $230 \mathrm{p}$.

INS. 2010. Institut national de la statistique, République du Niger.

Karim S, Mahamane A, Morou B, Saadou M. 2010. Dynamique de l'occupation des terres et caractéristiques de la végétation dans la Commune rurale de Simiri (région de Tillabéry, Niger). Annales de l'Université Abdou Moumouni, Tome XI-A, p. 166-177.

Ludwig JA, Wilcox BP, Breshears DD, Tongway DJ, Imeson AC. 2005. Vegetation patches and runoff-erosion as interacting ecohydrological process in semiarid landscapes. Ecology, 86(2): 288297. 
Mahamane A, Ichaou A, Ambouta JMK, Mahamane S, Morou B, Amani I, Mahamadou H, d'Herbès JM, Gineste P, Wata I, Issaka A. 2007. Indicateurs écologiques de la période optimale de remise en culture de jachères au Niger. Sécheresse, 18(4): 289-95.

Morou B. 2010. Impacts de l'occupation des sols sur l'habitat de la girafe au Niger et enjeux pour la sauvegarde du dernier troupeau de girafes de l'Afrique de l'Ouest. Thèse présentée à l'Université Abdou Moumouni de Niamey pour obtenir le titre de Docteur, 174p.

Moussa IB, Descroix L, Maiga OF, Gautier E, Adamou MM, Esteves M, Yéro KS, Abdou MM, Mamadou I, Breton EL, Abba B. 2011. Les changements d'usage des sols et leurs conséquences hydrogéomorphologiques sur un bassinversant endoréique sahélien. Sécheresse, 22: 13-24.

Philip MS. 2002. Measuring Trees and Forests ( $2^{\text {nd }}$ edn). CABI: London; 1-310.

Robert M. 1992. Le sol, ressource naturelle à préserver pour la production et l'environnement. Cahiers Agricultures, 1: 20-34.
Rondeux J. 1999. La Mesure des Peuplements Forestiers. Presses Agronomiques de Gembloux : Gembloux, Belgique.

Saadou M. 1990. La végétation des milieux drainés nigériens à l'Est du fleuve Niger. Thèse de Docteur ès Sciences Naturelles, Université de Niamey, Niger, 395p.

SAS Inc. 1999. SAS/STAT User's Guide. SAS Institute, Cary, NC.

SAS. 2010. Using JMP 9. SAS Institute Inc., Cary, NC, USA.

SUN. 2008. Méthode d'étude et d'analyse de la flore et de la végétation tropicale. Acte d'atelier sur l'harmonisation des méthodes, $74 \mathrm{p}$.

Yaméogo YT, Somé AN, Hien M. 2009. Etude préliminaire à une restauration de sols dégradés en zone soudanienne du Burkina Faso. Sécheresse, 20(1e): e32e38.

Zougmoré R, Zida Z, Kambou FN. 1999. Réhabilitation des sols dégradés: rôles des éléments nutritifs des plantes dans le succès des techniques de demi-lune et de zaï au sahel. Bulletin Erosion, 19: 536550. 